\title{
«Un ouvrage de référence pour tous»
}

Interview: Bruno Kesseli

Dr méd. et lic. phil., rédacteur en chef

Le compendium COMPACT marque depuis peu le retour sur le marché d'un ouvrage de référence sur les médicaments, publié sous forme de livre et déclinant tous les médicaments autorisés en Suisse. Dans une brève interview, Ulrich Schaefer, directeur de la société HCI Solutions, qui édite cet ouvrage, et Gert Printzen, membre du Comité central de la FMH, expliquent les raisons de ce nouveau lancement.

La forme imprimée du "Compendium des médicaments" a été suspendue en 2013. Trois ans plus tard, le compendium COMPACT est de nouveau lancé sur le marché, comme un ouvrage de référence sur les médicaments, publié sous forme de livre. Que signifie ce revirement?

Ulrich Schaefer: Après l'arrêt de la version imprimée obligatoire on pouvait supposer que le passage complet de la version papier à la version en ligne serait pratique et

«... les choses étaient claires pour nous: nous allions lancer une nouvelle version, mais compacte!»

rapide. En réalité, actuellement, des versions imprimées obsolètes du Compendium et du Bréviaire sont utilisées dans de nombreux endroits, cabinets médicaux, hôpitaux et centres de soins. Les sondages révélaient en outre que la version imprimée manquait à presque la moitié des médecins en cabinet. L'entreprise Zentiva se déclarant prête à prendre une grande partie des exemplaires, les choses étaient claires pour nous: nous allions lancer une nouvelle version, mais compacte!

Gert Printzen: Les sondages menés par la FMH révélaient un curieux phénomène: cette version imprimée intéressait certes les collègues d'un certain âge, mais du côté des jeunes, un besoin était également exprimé.

\section{Printzen, quel rôle a joué la FMH dans ce nouveau lancement? \\ D’une part, avant déjà, dans l'éditorial du Bulletin des mé- decins suisses [1], la FMH s'était «publiquement» exprimée sur cette question, générant un grand nombre de réponses de collègues exerçant en cabinet, qui nous demandaient d'intervenir en faveur du maintien du Compendium au format papier. D'autre part, nous avons abordé ce thème}

\section{Les personnes interviewées}

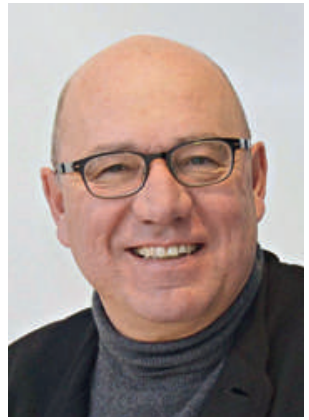

"Garantir un aperçu rapide»: Ulrich Schaefer sur le compendium COMPACT.
Ulrich Schaefer est pharmacien et a écrit des travaux dans le domaine de l'analyse informatique des principes actifs. Depuis 2011, il dirige I'entreprise $\mathrm{HCl}$ Solutions SA, qui regroupe les activités anciennement gérées par Documed, e-mediat et Triamun. Son travail est essentiellement axé sur la sécurité de la médication dans les systèmes intégrés. II est membre du Directoire de la IG-eHealth et d'eMediplan.

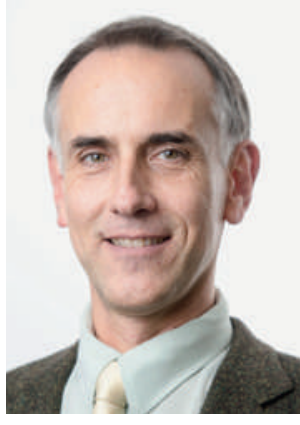

Identification d'un «besoin du côté des jeunes aussi»: Gert Printzen.
Gert Printzen est spécialiste en médecine de laboratoire et titulaire d'un diplôme universitaire de biochimie. Depuis 2010, il est membre du Comité central de la FMH, où il dirige les départements «eHealth - informatique et documentation médicale», "Produits thérapeutiques» et "Professions paramédicales». 
dans notre groupe de travail FMH eHealth, composé de représentants des organisations de base de la Chambre médicale, donc représentant leurs intérêts pour toute la Suisse. Enfin, au niveau politique, nous avons mis en place la Table ronde, afin de regrouper toutes les parties prenantes sur la question du Compendium des médicaments et de la sécurité des patients, et nous les avons convaincues que les données complètes et à jour devaient être organisées dans le Compendium. C'est la base de la version électronique, mais c'est aussi important pour la qualité au format papier.

\section{Comment se présente le concept du compendium COMPACT et en quoi se distingue-t-il de l'ancien Compendium des médicaments?}

Schaefer: Les dernières versions de l'«ancien» Compendium étaient de plus en plus encombrantes, à cause du volume de texte prescrit, et les monographies des différents médicaments couvraient plusieurs pages d'écriture serrée. Le nouveau compendium COMPACT contient les principales informations sur chaque médicament, rédigées sous une forme condensée par notre rédaction spécialisée, ce qui permet d'avoir un aperçu rapide.

Printzen: Il se peut que l'ancienne forme «longue» du Compendium manque à certains. Mais cette nouvelle forme fournit une première vue d'ensemble importante qui aidera à la prise de décision.

«Nous considérons ce nouvel ouvrage papier comme un complément optimal des platesformes numériques, à utiliser suivant les préférences et la situation.»

\section{Le Compendium est disponible sur Internet, compendium.ch. Savez-vous comment et par qui il est utilisé?}

Schaefer: Presque tous les professionnels du marché suisse de la santé utilisent la version en ligne et/ou les versions pour téléphones mobiles. Nos sondages réguliers nous permettent aussi d'avancer des chiffres: par exemple, $80 \%$ des médecins généralistes utilisent le site compendium.ch tous les jours.

\section{Quels groupes cibles touchez-vous avec le compen- dium COMPACT? S'agit-il de médecins d'un certain âge, qui sont moins à l'aise avec les outils de commu- nication électroniques modernes?}

Printzen: Comme nous l'avons mentionné au début de l'interview, l'éditorial du BMS a généré beaucoup de réactions directes. Même si le gros de la demande de maintenir la version imprimée du Compendium émanait plutôt de nos collègues d'un certain âge, le besoin

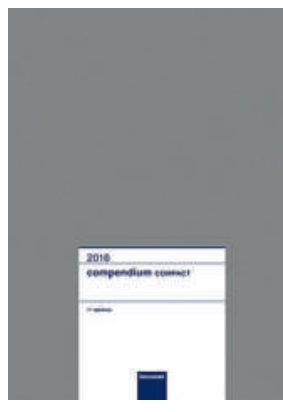

compendium COMPACT

Edition française

2016. 1022 pages. $145 \mathrm{CHF}$.

ISBN 978-3-906819-02-0

a aussi été exprimé par les plus jeunes. Eventuellement, nous ferons un jour un sondage à ce sujet.

Schaefer: C'est très clair: le compendium COMPACT est un ouvrage de référence pour tous. Il n'y pas que le numérique ou l'analogique. Nous considérons ce nouvel ouvrage papier comme un complément optimal des plates-formes numériques, à utiliser suivant les préférences et la situation.

\section{Dans quel contexte envisagez-vous l'utilisation du compendium COMPACT? Ses 1000 pages ne peuvent tenir dans la poche d'un veston...}

Schaefer: A chaque fois que c'est plus pratique...

Printzen: Notamment pendant les arrêts ou les blocages de la connexion Internet, ou lors de tout autre problème de transfert. Attendons de voir les retours que nous aurons concernant l'épaisseur et le nombre de pages de l'ouvrage.

Ne peut-on prévoir que bientôt les médias électroniques s'imposeront sur tout le territoire, dans le corps médical aussi? Envisagez-vous un avenir à long terme pour cette version imprimée?

Printzen: Les retours des publications de la FMH révèlent, selon moi, un besoin, même dans la génération alpha.

Schaefer: Il est certain que dans le cadre de la cybersanté, l'utilisation des médias électroniques va augmenter aussi dans le corps médical. Mais comme nous l'avons dit, les différents formats se complètent parfaitement. Plus que le support lui-même, ce qui est important, c'est de toute façon la fiabilité des contenus et la convivialité de leur présentation.

\section{Le Compendium des médicaments était distribué} directement et gratuitement à une grande partie du corps médical. Comment le compendium COMPACT arrive-t-il jusqu'aux médecins?

Schaefer: Contrairement à avant, il n'y a pas d'envoi de masse aux cabinets médicaux, hôpitaux et pharmacies. Le compendium COMPACT peut être commandé auprès des Editions médicales suisses EMH ou de la société Zentiva. 